\title{
Quantum Transmission in Disordered Insulators: Random Matrix Theory and Transverse Localization
}

\author{
Yshai AVISHAI ${ }^{(a, b)}$ and Jean-Louis $\operatorname{PICHARD}^{(a)}$ \\ (a) C.E.A., Service de Physique de l'État Condensé. \\ Centre d'Études de Saclay, 91191 Gif sur Yvette Cedex, France. \\ (b) Department of physics, Ben Gourion university, Beer Sheva, Israel. \\ and \\ Khandker A. MUTTALIB \\ Physics Department, University of Florida, Gainesville, Fl 32611, U.S.A.
}

\begin{abstract}
:
We consider quantum interferences of classically allowed or forbidden electronic trajectories in disordered dielectrics. Without assuming a directed path approximation, we represent a strongly disordered elastic scatterer by its transmission matrix $\mathbf{t}$. We recall how the eigenvalue distribution of $\mathbf{t} \cdot \mathbf{t}^{\dagger}$ can be obtained from a certain ansatz leading to a Coulomb gas analogy at a temperature $\beta^{-1}$ which depends on the system symmetries. We recall the consequences of this random matrix theory for quasi-1d insulators and we extend our study to microscopic three dimensional models in the presence of transverse localization. For cubes of size L, we find two regimes for the spectra of $\mathbf{t} . \mathbf{t}^{\dagger}$ as a function of the localization length $\xi$. For $L / \xi \approx 1-5$, the eigenvalue spacing distribution remains close to the Wigner surmise (eigenvalue repulsion). The usual orthogonal-unitary crossover is observed for large magnetic field change $\Delta B \approx \Phi_{0} / \xi^{2}$ where $\Phi_{0}$ denotes the flux quantum. This field reduces the conductance fluctuations and the average log-conductance (increase of $\xi$ ) and induces on a given sample large magneto-conductance fluctuations of typical magnitude similar to the sample to sample fluctuations (ergodic behaviour). When $\xi$ is of the order of the lattice spacing $(L / \xi \gg 5-6)$, the eigenvalue repulsion is weaker and the removal of a time reversal symmetry has a more negligible role. In those two regimes, the sample to sample fluctuations of the log-conductance are close to a normal one-parameter distribution, and $\xi^{-1}$ depends linearly on the disorder parameter. In these microscopic models, the Fermi energy dependence of the conductance is very similar to the one recently observed in small GaAs:Si wires.
\end{abstract}

Submitted for publication in Journal de Physique. 


\section{Résumé:}

Nous considérons les interférences quantiques de trajectoires electroniques classiquement permises ou interdites dans des diélectriques désordonnés. Sans faire une approximation de chemins dirigés, nous représentons un diffuseur élastique très désordonné par sa matrice de transmission $\mathbf{t}$. Nous rappelons comment la distribution des valeurs propres de $\mathbf{t} . \mathbf{t}^{\dagger}$ peut être obtenue à partir d'un certain ansatz conduisant à une analogie avec un gaz de Coulomb à une temperature $\beta^{-1}$ qui depend des symètries du système. Nous rappelons les conséquences de cette theorie de matrices aleatoires pour des isolants quasi-1d et nous étendons notre étude à des modèles microscopiques tridimensionnels où la localisation transverse est présente. Pour des cubes de taille $L$, suivant la valeur de la longueur de localisation $\xi$, nous trouvons deux regimes pour les spectres de $\mathbf{t} . \mathbf{t}^{\dagger}$. Quand $L / \xi \approx 1-5$, la distibution des espacements entre valeurs propres reste proche de celle de Wigner (repulsion des valeurs propres). Le cross-over habituel entre les cas orthogonal et unitaire a lieu pour un champ magnetique appliqué élevé $\Delta B \approx \Phi_{o} / \xi^{2}$ où $\Phi_{o}$ est le quantum de flux. Ce même champ réduit les fluctuations de conductance $g$ ainsi que la moyenne de $\log (g)$ (augmentation de $\xi$ ) et induit sur un echantillon donné de grandes fluctuations de la magnetoconductance d'une ampleur comparable à celles d'échantillon à échantillon (comportement ergodique). Quand $\xi$ est de l'ordre de la maille du réseau, $(L / \xi>>5-6)$, la répulsion des valeurs propres est plus faible et l'effet du champ magnetique cesse d'être important. Dans ces deux régimes, les fluctuations d'echantillon à echantillon de log $(g)$ sont bien décrites par une gaussienne à un paramètre et $\xi^{-1}$ varie linéairement en fonction du paramètre de désordre. Dans ces ces modèles microscopiques, la dependance en fonction de l'energie de Fermi de la conductance est très comparable à celle recemment observée sur de petits fils de GaAs:Si. 


\section{Introduction}

When one has to tackle the problem of electronic quantum transport, one of the usual microscopic starting points is the familiar Anderson tight-binding Hamiltonian:

$$
H=\sum_{i} \epsilon_{i} a_{i}^{\dagger} a_{i}+\sum_{<i, j>} h_{i j} a_{i}^{\dagger} a_{j}
$$

where the $\epsilon_{i}$ are the random site energies and the $h_{i j}$ represent the nearest neighbor couplings or transfer terms. For instance, the $\epsilon_{i}$ can be random with a rectangular distribution of width $W$ (Anderson model) or can take at random two values $\epsilon_{a}$ or $\epsilon_{b}$ (binary alloy model). The $h_{i j}$ can take for simplicity a uniform value $h$ if $i, j$ are nearest neighbors, 0 otherwise. Perturbations could be done when one of the two terms of $H$ (random substrate electrostatic potential or kinetic energy) is negligible compared to the other. The scaling theory of localization [1] assumes the existence of a universal $\beta(g)$-function of conductance $g$ relating those two limits through the mobility edge in three dimensions.

For disordered metals whose quantum conductance $g$, measured in unit of $e^{2} / h$, is larger than one, the conventional perturbation approach starts from plane-wave like states which are only weakly perturbed by the scattering caused by the random substrate $(W / h \ll 1)$. In this limit, electronic transport essentially results from quantum interferences of classically allowed diffusive paths and quantum theory is close to its semi-classical limit.

(i) We know for the three different possible symmetries (orthogonal, unitary and symplectic cases): the four loop order expansions [2] in $2+\epsilon$ dimensions of the scaling functions $\beta(g)$. One gets after integration the weak localization corrections to Boltzmann conductance, i.e. how $\langle g\rangle$ depends on the sample size $L$, the brackets denoting the ensemble average value.

(ii) The sample to sample conductance fluctuations around the ensemble average essentially satisfy gaussian distributions whose variance depends only on the basic symmetries and slightly on the dimensionality of the system [3] (Universal Conductance Fluctuations).

(iii) The conductance fluctuations induced on a given sample by the variation of an applied magnetic field or of the Fermi enegy are of similar magnitude [4] (ergodic behaviour). An applied magnetic field removes time reversal symmetry, suppressing the weak (anti) localization correction for $\langle g\rangle$ in the absence (in the presence) of spin-orbit scattering, halving the variance of the universal conductance fluctuations. The cross-over field is in general small in metals, corresponding to the application of a flux quantum $\Phi_{0}$ through the temperature dependent area where electrons keep their quantum coherence.

(iv) The conductance fluctuation yielded by a local reorganization of the substrate potential (single impurity move) have been understood mostly from known results on random walks, yielding a possible explanation for universal amplitude of observed $1 / f-$ noises [5].

For Anderson insulators, $W / h \gg 1$ and it is better to perturb the point-like-localized eigenstates of the random term in (1) by the kinetic transfer term. The perturbation removes the possible degeneracies of the $\epsilon_{i}$ (which is huge in the binary alloy model) and the eigenstates of (1) are still localized over domains of typical size $\xi$ which can extend over many impurities in the vicinity of the mobility edge. The Green function $G(E)$ between two sites $\mathrm{i}$ and $\mathrm{j}$ can be expanded in powers of the hopping integral $h$

$$
<i|G(E)| j>=\sum_{\Gamma} \prod_{i_{\Gamma}} \frac{h e^{i A}}{E-\epsilon_{i \Gamma}} .
$$


Here we have also introduced a magnetic vector potential $A$ for generality. The paths $\Gamma$ going from $\mathrm{i}$ to $\mathrm{j}$ are in this case a priori highly non classical, and electronic transport mainly results from classically forbiden trajectories which interfere due to the random sign of the denominator and to the vector potential. When all the factors $h /\left(E-\epsilon_{i}\right) \ll 1$, the shortest directed paths $\Gamma_{d}$ need only to be considered [6] and electron trajectories with self intersections have no significant contribution in this limit:

$$
<i|G(E)| j>\approx h^{L} \sum_{\Gamma_{d}} \prod_{i_{\Gamma_{d}}} \frac{e^{i A}}{E-\epsilon_{i}}
$$

The combinatorics of the shortest directed paths $\Gamma_{d}$ of length $L$ can be simply obtained if some cases (e.g if the sites $i$ and $j$ stand at the opposite corners of a square lattice). More generally, these directed paths relating two points separated by $L$ have transverse fluctuations extending over $(L \xi)^{\zeta}$. Assuming standard diffusion, one gets $\zeta=1 / 2$ but a more detailed analysis shows that dominant paths are "superdiffusive", yielding different values [7] for $\zeta$. One finds that:

(i) The localization length $\xi$ characterizing the decay of the propagator:

$$
<\log |<i| G(E)|j>|^{2}>\approx L\left(\rho-\xi_{0}^{-1}\right)
$$

depends on the disorder parameter $W$ through a local contribution $\xi_{0}=[\log (W / h)]^{-1}$ which comes from the exponentially small hopping term $[h / W]^{L}$ in (3) (binary alloy model with $\epsilon_{i}={ }_{-}^{+} W$ at $\left.E=0\right) . \rho_{0}$ is a global contribution embodying the $W$-independent quantum interferences of the directed paths in this particular model [8]. $\xi$ increases as a function of an applied field $B$ as $B^{1 / 2}$ with a universal factor, giving rise to a positive magnetoconductance [9]. This sign of the magnetoresistance is not reversed by the presence of a strong spin-orbit scattering [10], as it is in disordered metals.

(ii) The sample to sample conductance fluctuations are lognormally distributed, but with anomalous size dependence of the fluctuations. $\operatorname{var}(\log (g))=C . L^{2 \omega}$, where the prefactor $C$ does not depend on the mean $<\log (g)>\propto L, \omega=1 / 3$ in two dimensions and $\omega \approx 1 / 5$ in three dimensions [7-8]. The distribution of $\log (g)$ then contains more than a single parameter.

(iii) The application of a flux quantum $\Phi_{o}$ through the "cigare-shape domain", which contains the forward scatterered trajectories, yields a fluctuation $\delta(g) \approx g$ much smaller than the typical sample to sample fluctuations [11] (non ergodicity). No other characteristic magnetic field scale appears if self-intersecting trajectories are neglected.

(iv) The effect of the motion of a single scatterer has not been addressed in directed path theories. Broad distributions of the $\log$-conductance with a quasi-universal variance have been numerically observed without a directed path approximation [12].

The restriction of quantum interferences to this class of directed trajectories is usually made in ad hoc models (binary alloy distribution at an energy far from the $\epsilon_{i}$ ) where one assumes that the path contribution is always an exponentially decreasing function of its length. This does not take into account situations where small denominators could compensate the exponentially small value of $h^{L}$. Such quantum resonances necessarily occur in a device where carrier concentration can be varied by capacitive technics. This approximation does not describe also more general cases where transport involves complicated interferences between classically forbidden and allowed trajectories (vicinity of the mobility edge). In an Anderson model with a broad distribution of $h / \epsilon_{i}$, there is a 
finite probability to have clusters where the electron can diffuse easily, be backscattered and selfintersects its own trajectory before having to tunnel through large energy barriers. Such metallic clusters can yield non trivial magnetic field or spin-orbit scattering dependence in the vicinity of the mobility edge.

An alternative non perturbative theory for the analysis of quantum transmission in insulators is provided by a maximum entropy ansatz [13] using the transfer matrix $M$. This approach quantitatively describes quantum transmission through quasi-1d wires over length scale smaller or larger than their localization lengths, leading us to predict novel universal symmetry breaking effects [14]. We are interested to know its degree of validity outside quasi-one dimension in the presence of a strong transverse localization, and to compare its predictions from perturbation theory restricted to directed paths. We do not restrict ourself to the limit of an elementary localization of the conduction electrons around randomly located donors or acceptors, i.e. to a purely geometrical disorder, but we consider also systems closer to a mobility edge where localization results from complex interferences between classically allowed or forbiden trajectories. In this later case, it is likely that the relevant quantum interferences do not only involve simple forward scatterered directed paths, and that the effect of a strong spin-orbit coupling or of a large applied magnetic field have to be revisited $[15,16]$. Being outside a simple perturbative limit, we rely on numerical simulations which include all possible path contributions without any a priori simplification. We use as microscopic models either a random quantum wire network or an Anderson model with rectangular distribution of $\epsilon_{i}$.

This work belongs to a series of three papers devoted to quantum interferences in disordered insulators. One of them is theoretical [17] and analyzes how the purely quantum interferences which we consider are truncated by activated process at low temperature. The second [18] is experimental and gives the energy and magnetic field dependence of the conductance. One could then compare the results of our simulations to their results and note a striking (at least qualitative) similarity. The difficulty for a more quantitative comparison is due to the activated processes.

We recall in sections 2 and 3 the maximum entropy description of quantum transmission and its implications for quasi-one dimensional insulators (typical conductance and sample to sample fluctuations, symmetry breaking effects). We study in section 4 the modification of the physical constraint used in this maximum entropy description for three dimensional insulators. The persistence of the short range repulsion in presence of transverse localization is shown in section 5 through a study of the eigenvalue nearest spacing distribution of $\mathbf{t} . \mathbf{t}^{\dagger}$, though the long range interactions are weaker than usual random matrix behaviour (study of the spectral rigidity). In section 6 , the sample to sample fluctuations of $\ln (g)$ are studied in three dimensions for large disorder. We show that $\xi^{-1}$ varies as a function of the disorder parameter linearly from the mobility edge to the strongly localized regime. The effects of a variation of the applied magnetic field and of the Fermi energy are considered in section 7 and 8. We compare our results for the eigenvalues of $\mathbf{t} . \mathbf{t}^{\dagger}$ with those which are understood for the system Hamiltonian (section 9), and with the experimental results of Ref. 18 (section 10).

\section{Maximum entropy description of quantum scatterers}

We consider a $N$-channel quantum scatterer that we represent by its transfer matrix $M$. This matrix gives the electronic flux amplitudes at the right hand side of the scatterer 
in terms of the flux amplitudes standing at its left hand side. The statistical ensemble for a matrix $X$, related to $M$ by the relation

$$
X=\left(M^{\dagger} \cdot M+\left(M \cdot M^{\dagger}\right)^{-1}-\mathbf{1}\right) / 4,
$$

is chosen [13] as the most random matrix ensemble given the density $\sigma(\lambda)$ of the eigenvalues of $X$. This statistical ensemble of maximum information entropy for $X$ implies that the $X$-eigenvectors are statistically independent of the eigenvalues and are distributed totally at random. We denote by $\mathbf{t}$ the transmission matrix at the Fermi energy. Using a two-probe Landauer formula we express the conductance $g=2 . \operatorname{tr}\left(\mathbf{t} . \mathbf{t}^{\dagger}\right)$ as a linear statistics of the eigenvalues $T_{a}$ of $\mathbf{t} . \mathbf{t}^{\dagger}$ or the eigenvalues $\left\{\lambda_{a}\right\}$ of $X$ via the relations:

$$
g=2 \cdot \sum_{a=1}^{N} T_{a}=2 \cdot \sum_{a=1}^{N} \frac{1}{1+\lambda_{a}}
$$

the factor two coming from a possible spin or Kramer degeneracy. Sometimes, we refer to the eigenvalues $\left\{\lambda_{a}\right\}$ of $X$ as the radial parameters of $M$, since they can be also introduced through a natural "polar" decomposition of the transfer matrix.

The joint probability distribution $P\left(\left\{\lambda_{a}\right\}\right)$ can be easily calculated from this maximum entropy ensemble. One gets an expression formally identical to the Boltzmann weight of a set of classical charges free to move on a half line (the positive part of the real axis) at a well-defined "temperature" equal to $\beta^{-1}$ :

$$
P(\{\lambda\}) \propto \exp -\beta \cdot H\left(\left\{\lambda_{a}\right\}\right),
$$

and governed by an hamiltonian:

$$
H\left(\left\{\lambda_{a}\right\}\right)=-\sum_{a<b}^{N} \ln \left|\lambda_{a}-\lambda_{b}\right|+\sum_{a=1}^{N} V\left(\lambda_{a}\right) .
$$

The confining potential is given in terms of the input density $\sigma(\lambda)$ by

$$
V(\lambda)=\int_{0}^{\infty} \sigma\left(\lambda^{\prime}\right) \ln \left|\lambda-\lambda^{\prime}\right| d \lambda^{\prime}
$$

In usual random matrix theories, this is the classical Coulomb gas analogy which introduces a temperature, a one-body potential and a two-body interaction.

(i) The temperature is given by the fundamental symmetries which leave the system invariant (time reversal symmetry, spin rotation symmetry), $\beta=1,2,4$ respectively for the orthogonal, unitary and symplectic case. In the absence of spin-orbit scattering, $\beta=1$ without applied magnetic field and becomes equal to 2 if the applied magnetic field is large enough (removal of time reversal symmetry).

(ii) The confining potential $V(\lambda)$ can be adjusted in order to attract most of the $\lambda_{a}$ near the origin, yielding a large value for $g$ (metal), or on the contrary $V(\lambda)$ can be very weakly attracting, leaving the "charges" spread out along the positive real axis, yielding $g \ll 1$ (insulator). In other words, the spectrum of $X$ depends on the different physical parameters (Fermi momentum $k_{f}$, disorder measured by $l$, system size $L \ldots$ ) through a simple one-body potential $V(\lambda)$ in this maximum entropy ansatz.

(iii) As the value of $\beta$, the crucial logarithmic interaction between the $\lambda_{a}$ is obtained from the invariant measure of the space of matrices $X$ which are invariant under certain symmetries that each member of the statistical ensemble must satisfy. 
The consequences of the ansatz (8) on the spacing distribution of successive radial parameters and on their two point correlation functions have been carefully investigated [1920] for disordered conductors where the $\lambda_{a}$ are in part accumulated close to the origin. Detailed studies confirm both the presence of the infinite range logaritmic interactions and the change of temperature associated with symmetry breaking effects when the sample shape is nearly quasi-one dimensional. For higher dimensions $(d=2$ and 3$)$, the transverse diffusion slightly reduces the rigidity of the $\lambda$-spectra for high transmission or reflection (edges of the spectra), albeit the random matrix ansatz remains a good approximation.

In the quasi-1d limit, the ansatz (3) is consistent with a diffusion equation [13] which implies the quantitatively correct behaviours for the average and the variance of $g$ in the metallic limit, if the input density $\sigma(\lambda)$ satisfies a certain integro-differential equation. More important for the questions investigated here, this ansatz remains valid when the sample length is larger than the quasi-1d localization length, establishing the validity of this "Coulomb gas model" in the presence of longitudinal localization. In other words, the Coulomb gas model remains essentially stable with respect to matrix multiplication for a fixed number $N$ of channels.

\section{Longitudinal localization without transverse localization (quasi-1d limit)}

We summarize in this section previous results obtained from the global ansatz [13-21] and based on an analytic expression for $\sigma(\lambda)$ yielded from the diffusion equation [22] valid in the quasi-1d limit. The analysis is easier in terms of the variables $\left\{\nu_{a}\right\}$ related to the $\left\{\lambda_{a}\right\}$ through

$$
\lambda_{a}=\frac{\left(\cosh \left(\nu_{a}\right)-1\right)}{2} .
$$

In the large $N$-limit, one gets for the $\nu$-density

$$
\sigma(\nu)=\frac{2 l}{N L} \quad 0 \leq \nu \leq 2 L / l
$$

and zero elsewhere which gives for the confining potential:

$$
U(\nu)=\frac{N l}{2 L} \int_{0}^{2 L / l} \ln \left|\cosh (\nu)-\cosh \left(\nu^{\prime}\right)\right| d \nu^{\prime} \approx \frac{N \cdot l}{4 L} \nu^{2} .
$$

We write again the distribution $P\left(\left\{\nu_{a}\right\}\right)$ as a Boltzmann weight at a temperature $\beta^{-1}$ of a classical system, getting for the hamiltonian

$$
H\left(\left\{\nu_{a}\right\}\right)=-\sum_{a<b}^{N} V_{2}\left(\nu_{a}, \nu_{b}\right)+\sum_{c=1}^{N} V_{1}\left(\nu_{c}\right) .
$$

The two body interaction is now:

$$
V_{2}\left(\nu_{a}, \nu_{b}\right)=\ln \left|\cosh \left(\nu_{a}\right)-\cosh \left(\nu_{b}\right)\right|,
$$

and the one body potential contains a $\beta$-dependent term yielded by the change of variable:

$$
V_{1}\left(\nu_{c}\right)=-\frac{1}{\beta} \ln \left(\sinh \left(\nu_{c}\right)\right)+U\left(\nu_{c}\right)
$$


However, the quasi-1d localized limit is characterised by a particular behaviour of the spectral density: increasing the system size $L$ for a fixed number $N$ of channels, we end up to have:

$$
1 \ll \nu_{1} \ll \nu_{2} \ll \ldots \ll \nu_{N}
$$

which allowes us to simplify $V_{2}\left(\nu_{a}, \nu_{b}\right)$, since $\ln \left|\cosh \left(\nu_{a}\right)-\cosh \left(\nu_{b}\right)\right| \approx \max \left(\nu_{a}, \nu_{b}\right)$, to get approximately independent gaussian fluctuations of $\nu_{a}$ centered at

$$
\nu_{a}^{0}=\left(a-1+\frac{1}{\beta}\right) 2 L / N l
$$

and of variance $2 L /(\beta N l)$. Since $\nu_{1}=2 L / \xi$, we see that the localization length is proportional to $\beta$ :

$$
\xi=\beta N l \text {. }
$$

The $N$-dependence of $\xi$ is characteristic of the quasi-1d limit where tranverse localization is absent. Change of $\xi$ and change of the magnitude of the conductance fluctuations by a symmetry breaking effect are the two sides of the same phenomenon, since $\xi$ is defined from the ensemble average $\langle\log (g)>\approx 2 L / \xi$ and not from $\langle g\rangle$. The characteristic crossover fields reducing the magnitude of the conductance fluctuations and increasing $\xi$ in the absence of spin-orbit scattering are typically larger in insulators than in conductors. The change from orthogonal to unitary symmetry occurs when a flux quantum $\Phi_{0}$ is applied through the phase coherent domain in a conductor (temperature dependent) and through the localization domain for an insulator (temperature independent). The corresponding fields $B^{*}$ are of order of $\approx 10-100$ gauss in a conductor at very low temperature (a few $\mathrm{mK}$ ) and $\approx 0.5-2$ teslas for a large localization length insulator. For insulators with strong spin-orbit scattering, if one assumes that the removal of the Kramer degeneracy for $\nu_{1}$ by an applied magnetic field $B$ introduces a splitting which is negligible compared to the large value of $2 L / \xi$, the transition $\beta=4 \rightarrow \beta=2$ essentially halves the value of $\xi$. The existence of a positive magnetoresistance for insulators with strong spin-orbit scattering is in this theory the counterpart of the suppression of weak-anti-localization in conductors, in agreement with some experiments [16] and in disagreement with directed path theories [10].

The second important result that we have derived concerns the sample to sample fluctuations of the logarithm of the conductance since

$$
-\log (g) \approx 2 L / \xi=\nu_{1}
$$

in this quasi-1d insulating limit. We obtain a normal distribution of $\log (g)$ with

$$
\operatorname{var}(\log (g))=-<\log (g)>
$$

Note that contrary to approximations based on directed paths, $\log (g)$ has a one-parameter distribution.

In summary, the above results indicate that the validity of a "Coulomb gas model" for the $\left\{\lambda_{a}\right\}$ in the quasi-1d limit is not at all equivalent to the validity of Wigner-Dyson statistics since the spacings between successive $\lambda_{a}$ become so exponentially large that the two-body interaction is reduced to an effective one body potential. In particular, this density effect is responsible for the non-universality of $\operatorname{var}(g)$ in insulators. But one cannot check easily in this limit the underlying presence of the logarithmic interaction. 
If we consider now strongly disordered $L * L * L$ cubes, the increase of their size $L$ will increase the number $N=\left(k_{f} \cdot L\right)^{d-1}$ of channels in addition to the longitudinal length. Real $3 d$-insulators are then more likely than quasi- $1 d$ systems to be characterised by an exponentially large value of $\lambda_{1}$ in the large $L$-limit without having exponentially large intervals between successive $\left\{\lambda_{a}\right\}$. Does the Coulomb gas analogy which is at least an excellent approximation in the absence of tranverse localization, remains of some interest in a strong disorder limit? Do two successive $\lambda_{a}$ still repel each other when their spacing is not exponentially large, as implied by the ansatz? Does the coulomb gas "temperature" take the classical values implied by symmetry considerations? This is the first issue that we have to check in our numerical study.

\section{Density and confining potential in the presence of transverse localization (3d-limit)}

We have calculated series of $\left\{\lambda_{a}\right\}$ for different realizations of a microscopic model. We have used a $3 d$-network of disordered quantum wires as explained in appendix 1. Each link $(i, j)$ of this network is characterized by a one-dimensional uniform electrostatic potential $\epsilon_{i, j}$, which is taken at random with a rectangular distribution of width $W$. Real (propagating mode) or imaginary (decaying mode) wave-vector $k_{i, j}=\left(E-\epsilon_{i, j}\right)^{1 / 2}$ characterizes the electronic wavefunction along the link $(i, j)$. The matching conditions at each intersection satisfy the basic symmetry and conservation laws of quantum mechanics. We have alternatively used the Anderson model (1) with the same distribution for the random potential $\epsilon_{i}$, obtaining similar conclusions. (See also another recent numerical study of $3 d$ Anderson models by Markos and Kramer [23]).

In figure 1 , the density $\sigma(\nu)$ is given for $3 d$-cubes close to the mobility edge $(\mathrm{W}=4)$ and for larger disorder $(\mathrm{W}=7)$. Contrary to disordered conductors where this density is mainly concentrated near the origin [20], one can see that for large values of $W, \sigma(\nu)$ is negligible in the vicinity of the origin, indicating exponentially small transmission. In figure 2, we show the ensemble averaged positions $\left\langle\nu_{a}\right\rangle$ which are not widely separated for a dielectric cube of size $L$, contrary to the quasi-1d limit. One can note however that the spacings are larger near the lower edge of the spectra than in the bulk. We note that $g<1$ both for $W=4, \nu_{1} \approx 2$ and $W=7, \nu_{1} \approx 10$ when the system size $L=6$, but the study of the size dependence of the conductance $g(L)$ gives $\beta(g)=d \log (g) / d \log (L) \approx 0$ for $\mathrm{W}=4.5$ (mobility edge in the thermodynamic limit).

To complete the Coulomb gas model, one needs to know the one-body potential $V(\lambda)$. In figure $3, V(\lambda)$ obtained from an ensemble of numerically calculated series of $\left\{\lambda_{a}\right\}$ is given. Assuming the mean-field expression (5), we have directly performed the ensemble average of $\sum_{a=1}^{N} \ln \left|\lambda-\lambda_{a}\right|$, that we compare to an analytical fit whose the functional form is derived in appendix 2 :

$$
V(\lambda)=a \cdot \ln ^{2}\left(1+\frac{\lambda}{\lambda_{0}}\right) .
$$

The parameters $a$ and $b=1 / \lambda_{0}$ are adjusted to optimal values given in figure 3 .

\section{Short range repulsion of the transmission modes and sensitivity to an applied magnetic field.}

The Coulomb gas model implies that successive $\left\{\nu_{a}\right\}$ interact as:

$$
\ln \left|\cosh \left(\nu_{a}\right)-\cosh \left(\nu_{b}\right)\right| \approx \ln \left|\nu_{b}-\nu_{a}\right|
$$


if they are large and if their spacing is small. This logarithmic interaction must prevent small spacings (level repulsion) and the Wigner-surmise should give an approximate fit for the distribution of their spacing measured in average spacing units. We have studied this distribution, both for the first spacing $\left(\nu_{2}-\nu_{1}\right)$ and for spacings between $\nu$-levels in the bulk of the spectrum. As shown in figure 4, level repulsion persists even in the presence of transverse localization. For $\left\langle\nu_{1}\right\rangle \approx 2$ the obtained histogram is very close to the usual prediction of random matrix theory (Wigner surmise for $\beta=1$ ). This distribution also describes the spacings in the bulk of the spectrum (e. g. $\left.\nu_{7}-\nu_{6}\right)$ where the associated decay lengths $2 L / \nu_{a}$ are quite small. For larger disorder ( $W=7,<\nu_{1}>\approx 10$ ), level repulsion is weaker. The obtained histogram can then be fitted by an empirical formula proposed by Brody [24] (with $q=0.7$ )

$$
P_{q}(s)=A s^{q} \exp \left[-\alpha s^{q+1}\right]
$$

in order to interpolate from the correlated random matrix spectra (Wigner surmise $q=1$ ) to uncorrelated levels (Poisson distribution $q=0$ ). We can see in figure 2 that the mean spacing $\nu_{2}-\nu_{1} \approx 4$, which can partly explain the weakness of the repulsion $\left(\ln \left|\cosh \left(\nu_{2}\right)-\cosh \left(\nu_{1}\right)\right|\right.$ is more sensitive to the large value of $\nu_{2}$ than to the value of $\left.\nu_{2}-\nu_{1}\right)$. However, looking deeper in the spectrum, we can still note some deviation of the actual distribution of $\left(\nu_{7}-\nu_{6}\right)$ from the Wigner surmise (figure 5), while the mean spacing is much smaller. But the persistence of a strong eigenvalue repulsion between transmission modes with extremely short characteristic decay lengths $\left(\xi_{7}=2 L / \nu_{7} \approx 0.5\right)$ is by itself quite noticeable: the spacing distribution being much closer from a Wigner surmise than from a Poisson distribution characterizing uncorrelated eigenvalues.

Since we have approximately obtained spacing distributions in the presence of time reversal symmetry (orthogonal case) close to the Wigner surmise, it is interesting to know what is the value of the cross-over field $B^{*}$ needed for changing this distribution to the Wigner surmise characteristic of the unitary ensemble. It is also particularly interesting to see if $B^{*}$ characterises also the typical field scale where a change of $\xi$ occurs, in agreement with our theory relating the value $\xi$ to the importance of the fluctuation of the $\left\{\lambda_{a}\right\}$ which are controlled by the "temperature" $\beta^{-1}$. We have applied increasing magnetic field $B$ (measured in units of $\Phi_{o}$ per lattice cell).

We first note that weak fields (about one flux quantum through the sample) have no clear effects on the different $\left\langle\nu_{a}\right\rangle$ and on the spacing fluctuations. Near the mobility edge $(W=4)$, we observe as expected both the Wigner-surmise of the unitary ensemble $(\beta=2$, figure 6$)$ and a slight change of $\xi$ (increase by a factor $\approx 1.3)$ for $B=0.25$. One can see also in figure 6 that $P\left(\nu_{2}-\nu_{1}\right)$ does not depend very much on $B$ for larger disorder. We think that this is partly due to the gauge invariance of a lattice model which makes impossible to remove the coherent backscattering effects within too small localization domains. Two conclusions emerge from the magnetic field dependence of the spacing fluctuations. First, the cross-over field $B^{*}$ is much higher in insulators than in metals. Contrary to a metal where the typical electronic trajectories are random walks exploring the whole coherent sample, the typical trajectories in an insulator are enclosing much smaller areas characterized by much larger dephazing fields. Our results agree with the criterion $B^{*} \approx \Phi_{0} / \xi^{2}$. Second, the necessary field in order to significantly change $\xi$ is precisely the same which changes the spacing distribution, in agreement with the existence of a relation between the change of $\xi$ and the change of $\beta$. 
The study of the spacing distribution is not the more accurate test of the validity of the random matrix ansatz for $3 d$-insulators, probing more the short range eigenvalue repulsion than the long range spectral rigidity. The usual statistical measure of the long range rigidity is the $\Delta_{3}$-statistics introduced by Dyson and Mehta [25]. Once the spectra have been numerically unfolded [26] to series of constant averaged density, this statistics measure how the rescaled series deviate from a regular sequence as a function of the average number of considered levels. We show in figure 7 how the $\Delta_{3}$-statistics calculated from the $\left\{\lambda_{a}\right\}$ of a $3 d$ Anderson model deviates from the random matrix behaviour when $\xi \approx 1$. Similar behaviours characterize the network of random quantum wires previously considered.

The deviation of $P(S)$ and of the $\Delta_{3}$-statistics to the usual random matrix prediction can be partly due to the highly non-uniform density of the relevant variables $\left\{\lambda_{a}\right\}$ which could strongly weaken the effect of the logarithmic repulsion for the rescaled variables. In the quasi-1d limit for instance, we have shown that the ansatz (8) yields quasiindependent level fluctuations, giving a $P(S)$ or a $\Delta_{3}$-statistics closer to Poisson than to Wigner. In references 27, a crossover in $P(S)$ and in $\Delta_{3}$ from Wigner to Poisson is obtained within the random matrix framework, assuming infinite range logarithmic repulsion and a one-body potential qualitatively similar to Eq. 21. However, since we have shown for disordered conductors [20] that the $3 d$-value of the amplitude of the conductance fluctuations is contradictory with infinite range logarithmic repulsion for the $\left\{\lambda_{a}\right\}$, we speculate that the long range part of this repulsion must be even more severely damped by transverse localization than by transverse diffusion. But, from the study of those two microscopic models, we see that a short range repulsion persists in the presence of transverse localization. The shorter is $\xi$, the shorter is this range.

We note that a dimensionality dependent damping of the logarithmic repulsion has been shown [28] to be consistent with the known density-density correlations of Hamiltonian spectra in disordered conductors. Similarly, the long range part of the two body interaction in (8) may need to be modified in order to still write the distribution $P\left(\left\{\lambda_{a}\right\}\right)$ as the Gibbs distribution of a fictitious hamiltonian.

\section{Disorder dependence of the typical conductance and of its fluctuations}

In this section, we compare the actual behaviour of $\langle\ln (g)>$ and of its fluctuations to an extension of the results of section 3 and to the predictions implied by a direct path approximation [6-10]. We first note that contrary to the quasi- $1 d$ limit where $-\ln (g) \approx \nu_{1}$, $-\ln (g)$ is slightly larger than $\nu_{1}$ in three dimensions $\left(\nu_{2}, \nu_{3}, \ldots\right.$ weakly contribute to $\left.\sum_{a=1}^{N}\left(\cosh \left(\nu_{a}\right)-2\right)^{-1}\right)$. Extracting the localization length from the size dependence of $<\ln (g)>$ and locating the critical value $W_{c}$ from the criterion $d \ln (g) / d \ln (L)=0$ we obtain (figure 8) a simple linear dependence:

$$
\xi^{-1}(W) \propto\left(W-W_{c}\right)
$$

of the inverse localization length for the $3 d$-network of quantum wires. In the vicinity of the critical point, this linear law is consistent with the value reported in the experimental litterature for the critical exponent, but lower than most of the other numerical results given by finite size scaling studies of various microscopic models (for a review, see Ref. 29). But we mainly point out in this study that this linear dependence persists when the localization length reduces to the lattice spacing, without showing a logarithmic behaviour 
that one could naively guess from the length dependence $h^{L}$ of the transfer term in a directed path approximation (Eq. 4).

Considering the sample to sample fluctuations of $\ln (g)$, we have obtained histograms in rough agreement with a normal distribution of the $\log$-conductance. The variance of $\ln (g)$ varies as a function of the disorder and of system size such that relation (20) continue to be observed (figure 9) in three dimensions. A similar result have been noticed in the $2 d$-localized regime [21] and is consistent with one-parameter scaling, and inconsistent with directed path theories for the investigated range of parameters. This one-parameter law is observed in different microscopic models (Anderson model and network of random quantum wires). The comparison between the "random matrix prediction" and the directed path result can be sligthly biased since we consider the side to side transmission through a disorder cube, and not how electrons are transmitted from a corner to the opposite corner of a cube. We have noted also that the equality between the mean and the variance of $\nu_{1}$ is even more accurate than for $\ln (g)$.

\section{Magnetoconductance Fluctuations and Sample to Sample Fluctuations.}

In disordered conductors, a magnetic field $B . L^{2} \approx \Phi_{0}$ removes time-reversal symmetry (cross-over from the orthogonal ensemble to the unitary ensemble), suppressing weaklocalization corrections and halving the variance of the universal conductance fluctuations. A (typically weak) magnetic field change of this order induces also magnetoconductance fluctuations of magnitude equal (to first order in $1 / g$ ) to the sample to sample fluctuations under applied magnetic field [4]. We have already noticed [15] in two dimensions that this ergodicity remains essentially correct in the localized regime for (large) field change $\Delta B \approx B^{*}=\Phi_{0} / \xi^{2}$, at least when $-\log (g) \approx 2-7$. This "ergodic" behavior was proposed for explaining the large reproducible magnetoconductance fluctuations observed in the Mott hopping regime even for macroscopic size insulators $(L \approx 1 \mathrm{~mm})$. It is then interesting to compare in three dimensions the typical magnitude of the magnetoconductance fluctuations on a given sample to the sample to sample fluctuations at given field. We do not mean here a rigorous statement about possible exact ergodicity in insulators but a mere comparison of the typical orders of magnitude. Due to the gauge invariance of our system under change of applied flux through lattice cell by $\Phi_{o}$ and due to the large value of $B^{*}$ in insulators, a large statistics of those magnetofluctuations cannot be obtained.

In Figure 10, we show how the first $\left\{\nu_{a}\right\}(a=1,2, \ldots, 5)$ fluctuate for a given sample with $W=5.5$ as a function of $B$. The fluctuations of $\nu_{1}$ essentially agree with an ergodic behaviour, as illustrated in figure 11. In addition to the large ergodic fluctuations induced by $\Delta B \approx B^{*}$, one can notice smaller non ergodic fluctuations which characterizes also the other $\nu_{a}$ deeper in the spectrum (figure 10) or $\nu_{1}$ itself for larger disorder (figure 12). We note that larger disorder increases the scale $B^{*}$ of the "ergodic" fluctuations and prevents us to observe them when $\nu_{1} \gg 1$, leaving only smaller fluctuations of characteristic field scale which does not seem to depend on $W$.

We point out that $B^{*}$ in a conductor is temperature dependent (the relevant area being the coherent domain), while $B^{*}$ in an insulator corresponds to applying a flux $\approx \Phi_{0}$ through a temperature independent localization domain typically smaller than the coherence length $\approx L_{M o t t}$ in an activated regime.

\section{Effect of a variation of the Fermi energy}


Conductance fluctuations can be observed in insulating wires where the carrier concentration can be controlled by a gate potential, as shown in Ref. 18. It is interesting to compare the "gate"-induced fluctuations observed at very low temperature with the Fermi energy dependence of $g$ occuring in our microscopic model. Moreover, this energy dependence provides interesting information about characteristic times of the transmission processes. The simpler case is an isolated resonance describable by a Breit-Wigner formula [30]:

$$
g(E) \propto T(E) \approx \frac{\left(\Gamma_{e} / 2\right)^{2}}{\left(E-E_{r}\right)^{2}+\left(\Gamma_{e} / 2\right)^{2}}
$$

where $E_{r}$ is the energy of the resonant state and the inverse of the resonance width $\Gamma_{e}$ gives the (long) time spent by the electron trapped in this resonant localized state. In more complex cases where resonances and direct processes are mixed, a smooth energy dependence of the transmission fluctuations still corresponds to predominant fast direct processes, while short energy scale fluctuations are related to slow resonant transmission, a priori neglected in a directed path approximation. In figures 13.a and 13.b, one can see how the "gate"-induced fluctuations are different when $g \approx 1$ (mobility edge, roughly symmetric "normal" fluctuations) and when the system is deeper in the localized regime (a few resonant peaks superimposed on a smooth exponentially small background). Both in the experiments reported in Ref. 18 and here, these peaks can be fitted by the BreitWigner formula (25). Of course in the experiments, inelastic processes must in general be taken into account [17]. In figure 14, we can see how the applied magnetic field modifies this resonant behaviour. When there is a resonant peak, a variation of $B$ detunes the resonance from its $B=0$ value, inducing large conductance fluctuations around that peak. On the contrary, far from the resonances, in the "valleys", the $B$-dependence is much weaker. This leads us to distinguish two effects of a variation of $B$. A (small) orbital effect which modifies the quantum interferences of the shortest directed paths which controlled a long range hop without intermediary resonances and a large effect resulting from the shifts of the resonant peaks. When the sample to sample fluctuations are studied, these two efects are mixed, though the probability to have a tuned resonance in a sample taken at random is very small.

A more systematic study of the Wigner time delay matrix will be reported in a future publication for separating the effects of the long resonances from the directed path contribution.

\section{Comparison with level statistics of the Hamiltonian matrix.}

In this study, we have investigated the validity of a (too) simple maximum entropy description of the transfer matrix in the limit of extreme (transverse) localization. In this description, the logarithmic interaction between eigenvalues and the value of $\beta$ are related to a good randomization of the eigenvectors. Therefore, such a description is not appropriate for matrices whose eigenvector distribution undergoes important change as a function of the system parameters (e.g. eigenvector localization).

For the hamiltonian matrix, the understanding which emerges from recent studies [28, 31] can be summarized as follows in three dimensions. The logarithmic repulsion remains valid for a $N \propto L$ nearest neighbour levels in the metallic phase, for a cube of size $L^{3}$. In the localized phase, some level repulsion is still observed for small $L$, but is expected to disappear in the thermodynamic limit where the levels would be totally uncorrelated as 
those of hamiltonian (1) in the absence of hopping terms. One can argue that there is a poor overlap of the wave fuctions due to exponential localization, such that levels mainly depend on spatially disconnected part of the random substrate. Therefore levels are less and less correlated for large $L$ in the absence of long-range correlation of the random variable $\epsilon_{i}$ in (1). For finite size, one has intermediary cases where the spacing distribution $P(S)$ differs from the Wigner surmise more or less as in figure 4, and the deviations scale with $L$ according to usual finite size scaling. The mobility edge is characterized [32] by a novel scale invariant $P(S)$.

It is then clear that, in the case of the hamiltonian matrix, "classical" random matrix theory with eigenvector distribution invariant under orthogonal, unitary or symplectic transformations and without eigenvector-eigenvalue correlations have to be extended to more complicated theories. Banded matrices with independent entries could be more realistic for describing hamiltonians in the quasi-1d limit, but very few analytical results are available for their eigenvalue distributions [33].

The random matrix $X$ differs from the hamiltonian matrix. Localization appears in the presence of exponentially large eigenvalues $\left\{\lambda_{a}\right\}$. Our simple maximum entropy approach contains, via the average density $\sigma(\lambda)$, some information on the localization of the wavefunctions, contrary to a similar approach for the hamiltonian matrix. One can then expect a better approximation of spectral correlation functions from this type of approach for $X$ than for $H$. Nevertheless, a numerical study of the $X$-eigenvectors and of the scale dependence of $P(S)$ in the localized regime will be very useful. The persistence of level repulsion for $X$ is associated with the spatial overlap of electronic paths associated with neighboring channels. This overlap can be partly related to the importance of resonant tunnelling through a few localized states. Electronic modes described by the first few $\nu_{a}$ would be essentially modes where the electrons would be trapped during a long time in overlapping sets of certain favorable resonant states before being transmitted, as indicated by the large energy fluctuations shown in section 6 . Single scatterer motions in those sensitive regions would induce correlated fluctuations of this set of $\nu_{a}$, as observed in Ref. 12. On the contrary, in a situation where shortest directed paths are predominant, one can imagine that the modes of transmission from on side to the opposite side of a large cube are associated to different non intersecting paths and are essentially uncorrelated.

\section{Comparison with experiments and activated transport.}

A comparison between random matrix description, directed path theory, numerical simulations and low temperature experiments has to be done with great care. We consider in this work quantum transmission without activated process and we do not a priori neglect the important phenomenon of resonant tunneling which could be more or less truncated at finite temperature by activated hopping. We refer for this to the join paper by Bouchaud and Ladieu [17], where the cross-over between quantum coherent transmission and activated transport is considered. Let us mention the physical argument implicitely behind the directed path approximation: if transport is not entirely coherent, but partly activated, one is precisely interested by long quantum hop between nearly resonant states, without additional resonances between them. In other words, the lifetime of an elastic resonance could be too long compare to the phase-breaking time at finite temperature. Then the propagator evaluated in directed path approximations is implicitely related to activated transport and might not necessarily describe zero temperature elastic 
quantum transmission. In one dimension for instance, Azbel has pointed out the role of resonances [34] and shown clearly that the shortest chains have not necessarily the best transmission ( a longer chain with a tuned resonance can be more transparent for the electrons than a shorter one).

When the localization length is very short (of order of the Bohr radius of donor or acceptor impurities), we are dealing with an "usual" bounded state and long range quantum transmission over $L_{M o t t}$ is restricted to interferences between forward scattered paths in a "cigare shape" domain of longitudinal length $L_{\text {Mott }}$ and of tranverse length $\left(L_{M o t t} \cdot \xi\right)^{\zeta}$. Those directed hops are characterized by a relatively short time. When the localization length is longer, we are dealing with a more complicated Anderson insulator, and one can question if the long time elastic transmission that we study in this work can occur between successive inelastic collisions.

However, despite the difficulty to take into account activated process and complicated quantum resonances, an element of answer is given by some striking similarities between the energy and magnetic field dependence of the conductance of our zero temperature microscopic model and the behaviours observed by Ladieu, Mailly, and Sanquer on a Si:GaAs wire at very low temperature, as illustrated in figure 15.

\section{Appendix 1: Microscopic $3 d$-model of disordered quantum wire networks}

The studied microscopic model can be considered as a system of narrow wave guides which intersect each other. We take the limit in which the transverse size of each wave guide tends to zero so that the motion between intersections is virtually one dimensional. In this limit, there is no underlying simple Hamiltonian. The mathematical foundation of the model has been studied by Exner and Seba [35]. The basic symmetry and conservation laws of quantum mechanics determine the motion completely once the matching conditions at each intersection are specified. Similar concepts have been used by Avron and coworkers in their study of adiabatic networks [36]. For simplicity of presentation the number of space dimensions is fixed at $d=2$ for the illustrative part (figures etc). Our numerical calculations are however carried out for a three dimensional network $d=3$. Consider then a square lattice of one dimensional wires with lattice spacing $a$. We will denote by $L$ the longitudal dimension of the system and by $N$ its transverse dimension (our numerical calculations are performed on cubes with $L=N)$. On this lattice of wires an electron can move subject to the laws of quantum mechanics. In the most general case there is a wave

number $k_{j}$ on each bond $j$ of the lattice. We have $k_{j}=\sqrt{E_{f}-v_{j}}$ where $v_{j}$ are random numbers drawn from a rectangular probability of width $W$. If $W>E_{f}$, some of the $k_{j}$ are purely imaginary and there is a disordered combination of plane-wave propagation and tunneling. In Fig. (16) we depict such a finite lattice. A site can therefore be labeled by a pair of numbers $(\ell, n), \ell=1,2, \ldots, L ; n=1,2, \ldots, N$. An electron can travel freely on each bond (wire) subject to the Schrodinger equation. We will employ the index $\mathrm{j}$ for a general bond (horizontal or perpendicular) and $\mathrm{n}$ for horizontal bonds only. Thus, if the wave number on a certain bond $\mathrm{j}(\mathrm{j}=1,2, . . \mathrm{LN})$ is $k_{j}$, the electron's wave function on this bond is a linear combination of plane waves

$$
\Psi_{j}=a_{j} e^{i k_{j} x}+b_{j} e^{-i k_{j} x}
$$

where for horizontal (vertical) bonds, the coordinate $\mathrm{x}$ is measured from the left (down) site. Imagine now that the $N$ sites on the left of the lattice (these are the sites $(1, n))$ and 
the $N$ sites on the right of the lattice (these are the sites $(L, n)$ ) are connected to electron reservoirs by free wires, namely, the electron's wave number on these wires is the Fermi wave number $k_{F}$. Then we have a quantum mechanical system whose conductance $g$ (at zero temperature) can be defined in the following way. Let an incoming wave $e^{i k x}$ of unit amplitude reach a site $(1, n)$ on the left column of the lattice. Then the wave function at the $m^{t h}$ exit wire on the right (namely to the right of site $(L, m)$ ) will be $t_{m n} e^{i k x}$ where $t_{m n}$ are the elements of the complex transmission matrix $t$. Likewise, the wave function at the $m^{t h}$ exit wire on the left (namely to the left of site $(1, m)$ ) will be $r_{m n} e^{-i k x}$ where $r_{m n}$ are the elements of the complex reflection matrix $r$. Unitarity (current conservation) implies the equality

$$
t t^{\dagger}+r r^{\dagger}=t^{\dagger} t+r^{\dagger} r=I_{N},
$$

where $I_{N}$ is the unit $\mathrm{NxN}$ matrix. The linear response theory now relates $g$ to the transmission coefficients in a simple way. If we consider this system as a collection of random resistances we notice that, unlike the classical situation in which conductances combine rationally to one another (either in parallel or in series) the situation here is much more complicated, since at each intersection the wave function must be matched according to the rules of continuity and current conservation. Let $\Psi_{1}, \Psi_{2}, \Psi_{3}$ and $\Psi_{4}$ be the wave functions of the electron in the four links connected by a given site. The four respective wave numbers are denoted by $k_{1}, k_{2}, k_{3}$ and $k_{4}$ respectively. Then at this site, the following continuity equation should be satisfied,

$$
\Psi_{1}=\Psi_{2}=\Psi_{3}=\Psi_{4}
$$

The relation between derivatives at the intersection is somewhat arbitrary as long as it is compatible with current conservation. For simplicity and consistence with previous works [37-38] we will assume the following relation,

$$
\Psi_{1}^{\prime}+\Psi_{2}^{\prime}=\Psi_{3}^{\prime}+\Psi_{4}^{\prime}
$$

where, following Eq. (26),

$$
\Psi_{j}^{\prime}=k_{j}\left(a_{j} e^{i k_{j} x}-b_{j} e^{-i k_{j} x}\right) .
$$

These relations give four equations relating the coefficients $a_{j}, b_{j}$ of Eq. 26 of the plane waves on each of the four links (see Fig. 16). The most natural algorithm by which one can evaluate the conductance is the transfer matrix method. Here, however, we intend to study the system also in the far insulating regime. A straightforward transfer matrix approach is hence numerically dangerous since the product of many matrices may blow up. Furthermore, we intend to study a cubic system with $L$ up to 12 . For a $3 d$-cube $(N=L)$ the size of the (complex) transfer matrix will then be $2 N^{2}=288$, which is quite large. It is then indispensable to invent a method which does not rely on a (possibly divergent) product of large matrices and at the same time will employ matrices of smaller size. This method is explained below in a heuristic manner. Its rigorous justification goes back to the theory of wave guides. It involves the algebra of transmission and reflection matrices whose size is $N^{2}$. Recently it has been adapted for studying conductances in the insulating regime [38]. The disadvantage here is that each step requires an inversion procedure instead of a simple multiplication as is required by the transfer matrix algorithm. The advantage is that the transmission and reflection matrices are bounded through the unitarity relation and hence there is no danger of spurious exponential Lyapunov divergence. 
Consider then a wave approaching a system of two barriers " 1 " and " 2 ". The transmission and reflection amplitudes for each individual barrier $i(i=1,2)$, independent of the other one, are assumed to be given by $t_{i}$ and $r_{i}$ if the wave approaches the barrier from the left and by $t_{i}^{\prime}$ and $r_{i}^{\prime}$ if the wave approaches the barrier from the right. For a $N-$ channel problem, the transmission and reflection amplitudes are $N * N$-complex matrices. They are usually arranged in an $S$-matrix pertaining to barrier $i$,

$$
S_{i}=\left(\begin{array}{cc}
t_{i} & r_{i}^{\prime} \\
r_{i} & t_{i}^{\prime}
\end{array}\right), i=1,2
$$

The $S$-matrix is unitary: $S i^{\dagger} S i=I, S i S i^{\dagger}=I$ (the unit matrix in the appropriate space). The transmission and reflection amplitudes pertaining to the combined system are $t_{12}, r_{12}$ $t_{12}^{\prime}$ and $r_{12}^{\prime}$. Our task is then to express these four amplitudes in terms of the amplitudes of the individual channels. These relations are given explicitly as follows,

$$
\begin{gathered}
t_{12}=t_{2}\left(1-r_{1}^{\prime} r_{2}\right)^{-1} t_{1} \\
r_{12}=r_{1}+t_{1}^{\prime} r_{2}\left(1-r_{1}^{\prime} r_{2}\right)^{-1} t_{1} \\
t_{12}^{\prime}=t_{1}^{\prime}\left(1-r_{2} r_{1}^{\prime}\right)^{-1} t_{2}^{\prime} \\
r_{12}^{\prime}=r_{2}^{\prime}+t_{2} r_{1}^{\prime}\left(1-r_{2} r_{1}^{\prime}\right)^{-1} t_{2}^{\prime} .
\end{gathered}
$$

In terms of the $\mathrm{S}$ matrices we then write

$$
S_{12}=S_{1} * S_{2}
$$

where the star product operation defined through Eqs. (32 - 36) has been applied by Redheffer in the theory of waveguides [39]. It is then possible to add the barriers one after the other until the entire system is worked out. The unitarity bound on the reflection and transmission matrices assures the existence of the inverse $\left(1-r_{1}^{\prime} r_{2}\right)^{-1}$ and guarantees the convergence of the procedure for any number of steps. It is worth mentioning here that if the barriers are infinitesimally close to each other, the star product is transformed into a non linear differential relation which eventually leads to a matrix Riccati equation for the reflection amplitudes.

We have thus explained how the transmissions of individual units are combined to give the transmission of the whole system. Now we have to explain what are the pertinent units and how the transmission and reflection through each unit is computed. Transmission and reflection occur at each column of sites followed by the accumulation of phases as the wave propagates between two such columns. Thus, each unit consists of a column of sites together with the links located on its right side (see Fig. 16). In order to evaluate the transmission and reflection at a given column of sites we number the sites from 1 to $N$, and consider a site $m(m=1,2, \ldots N)$. The wave number to its left is $k_{m}$ while the wave number to its right is $p_{m}$. The wave numbers in the transverse direction are $q_{m}$ (above the site) and $q_{m-1}$ (below the site). We adopt here periodic transverse boundary conditions so that $q_{0}=q_{N}$. On a transverse link between sites $m$ and $m+1$ the wave function is given by

$$
\Psi(y)=c_{m} e^{i q_{m} y}+d_{m} e^{-i q_{m} y}
$$

where the coordinate $y$ is measured from link $m$. An incoming wave of unit amplitude reaching site $n$ from the left will provoke, in any site $m$, leftward reflected waves with 
reflection amplitude $r_{m n}$, as well as rightward transmitted waves with transmission amplitude $t_{m n}$. The matching equations [Eqs. (28-29)] now imply the following relations (the dependence of the transverse coefficients $c$ and $d$ on the incoming index $n$ will not be indicated.

$$
\begin{gathered}
\delta_{m n}+r_{m n}=t_{m n}=c_{m}+d_{m}=c_{m-1} e^{i q_{m-1} a}+d_{m-1} e^{-i q_{m-1} a}, \\
k_{m}\left(\delta_{m n}-r_{m n}\right)+q_{m-1}\left(c_{m-1} e^{i q_{m-1} a}-d_{m-1} e^{-i q_{m-1} a}\right)=p_{m} t_{m n}+q_{m}\left(c_{m}-d_{m}\right),
\end{gathered}
$$

where $\delta_{m n}$ is the Kronecker delta function. The equality $\delta_{m n}+r_{m n}=t_{m n}$ results simply since reflection and transmission occur at the same point in space. From the first set of equations (39) one can now eliminate the coefficients of the transverse motions pair by pair using the relations

$$
\begin{gathered}
c_{m}+d_{m}=t_{m n} \\
c_{m} e^{i q_{m} a}+d_{m} e^{-i q_{m} a}=t_{m+1, n} .
\end{gathered}
$$

We now substitute the solution of equations (40 - 41) into equation (39) and replace $r_{m n}$ by $t_{m n}-\delta_{m n}$. As a result we get a set of equations for the transmission matrix $t=t_{m n}$ of the pertinent site of columns which reads

$$
A t=2 K
$$

where the $N * N$-matrices $A$ and $K$ are given by

$$
\begin{gathered}
A(m, m)=k_{m}+p_{m}+i\left[q_{m} \cot \left(q_{m} a\right)+q_{m-1} \cot \left(q_{m-1} a\right)\right] \\
A(m, m+1)=-i q_{m} \csc \left(q_{m} a\right) \\
A(m, m-1)=-i q_{m-1} \csc \left(q_{m-1} a\right) \\
K=\operatorname{diag}\left(k_{1}, k_{2}, \ldots k_{N}\right)
\end{gathered}
$$

with $m=1,2, \ldots, N$ and $N+1$ or 0 are defined modulo $N$. The solution of Eq. (42) yields the transmission matrix from left to right and then the corresponding left to left reflection matrix $r=t-I_{N}$ ( $I_{N}$ is the unit matrix) is obtained. To get the matrices pertaining to waves approaching from the right, one has to interchange the roles of $k_{m}$ and $p_{m}$. Since the matrix $A$ is not affected by this swap, it does not require any additional matrix inversion procedure. It is worthwhile noticing here that in the presence of a constant magnetic field $B$ perpendicular to the direction of propagation the only change in the above formalism concerns the non diagonal elements of the matrix $A$. If the columns of sites are numbered as $1,2, \ldots, L$, and the magnetic flux per plaquette is $\phi=B a^{2}$ in units of $\left.\Phi_{0}\right)$ then at column $\ell(\ell=1,2, \ldots, L)$ one has

$$
\begin{aligned}
& A(m, m+1) \rightarrow A(m, m+1) e^{2 \pi i \ell \phi}, \\
& A(m, m-1) \rightarrow A(m, m-1) e^{-2 \pi i \ell \phi} .
\end{aligned}
$$

These phases are identical to those multiplying the hopping integrals (the Peierls substitution) used e.g in tight binding models. What is left in order to complete the calculations of the individual unit shown in Fig. (16) is to take account of the phase between two units. This is easily accomplished using the matrix $\mathrm{P}=\operatorname{diag}\left(p_{1}, p_{2}, \ldots p_{N}\right)$ and the substitutions

$$
t \rightarrow e^{i P} t, r \rightarrow r, t^{\prime} \rightarrow t^{\prime} e^{i P}, r^{\prime} \rightarrow e^{i P} r^{\prime} e^{i P}
$$


We have thus explained how to evaluate the transmission and reflection amplitudes for each individual unit in the random link model. Together with the composition law explained in the first part of this section (Eqs. (33 - 36)) we have at hand an algorithm for a numerical study of the full system. We repeat here that our approach will mostly be used in three dimensions, for which the relevant equations are slightly more complicated.

The radial parameters $\left\{\lambda_{a}\right\}$ are then obtained from the eigenvalues $T_{a}$ of $\mathbf{t} . \mathbf{t}^{\dagger}$ using $T_{a}=1 /\left(1+\lambda_{a}\right)$.

\section{Appendix 2. Functional form of the analytical fit for $V(\lambda)$}

Although the actual density $\sigma(\nu)$ differs from a simple uniform density between two nonzero values, it is interesting to to investigate what $V(\lambda)$ is yielded from this crude approximation (a uniform density for the $\nu$-variable between two strictly positive values $2 L / A$ and $2 L / B)$. One gets:

$$
U(\nu)=C \cdot \int_{2 L / A}^{2 L / B} \ln \left|\cosh (\nu)-\cosh \left(\nu^{\prime}\right)\right| d \nu^{\prime}
$$

where $C=(N / 2 L)(1 / B-1 / A)^{-1}$.

The derivative is given by:

$$
\frac{\partial}{\partial \nu} U(\nu)=C \cdot \ln \left|\left(\frac{\exp (2 L / A)-\exp (\nu)}{\exp (2 L / A)-\exp -(\nu)}\right) \cdot\left(\frac{\exp (2 L / B)-\exp -(\nu)}{\exp (2 L / B)-\exp (\nu)}\right)\right|
$$

which presents spurious divergences at the band edges $2 L / A$ and $2 L / B$. Ignoring those divergences and after integration, one can see that the corresponding $U(\nu)$ is characterized in presence of transverse localization $(A \neq 0)$ by a flat part for $0 \leq \nu \leq \nu_{0} \approx 2 L / A$ and by a quadratic increases $\propto\left(\nu-\nu_{0}\right)^{2}$ for $\nu_{0} \leq \nu$. Coming back to the original variable $\lambda$, we then expect a confining potential increasing as $\left(\ln (\lambda)-\ln \left(\lambda_{0}\right)\right)^{2}$ for $\lambda \gg \lambda_{0} \gg 1$ and presenting a flat part for $\lambda \leq \lambda_{0}$. This suggests that we try to fit the $V(\lambda)$ calculated from a microscopic $3 d$-model assuming relation (5) with the following analytical expression

$$
V(\lambda)=a \cdot \ln ^{2}\left(1+\frac{\lambda}{\lambda_{0}}\right)
$$

The agreement is good for small enough values of $\lambda$, as shown in figure 3 . It is worth to notice that the validity of this fit seems very general: it has been checked in $2 d$ and $3 d$ disordered metals modelled either by random quantum wire networks or by tight-binding Anderson hamiltonians [20].

\section{Acknowledgements}

J.L.P. has benefited from useful discussions with the other members of the 1991program on "mesoscopic system" at the Institute for Theoretical Physics at Santa Barbara and with the members of the E.E.C. Science program Contract No. SCC-CT90-0020 "Localization and Transport Fluctuations in Microstructures", notably B. Kramer and P. Markos. We acknowledge also extensive discussions with J.P. Bouchaud, F. Ladieu and M. Sanquer. Part of this work at University of Florida was supported by N.S.F. grant no. DMR-8813402. 


\section{Figures Captions}

Figure 1: Average density $\sigma(\nu)$ calculated for cubes of size $L=6$ for disorder parameters $W=4$ (circles) and $W=7$ (squares).

Figure 2: Ensemble averaged $\left\langle\nu_{n}\right\rangle, n=1, \ldots, L^{2}$ for the same cubes as in Fig. 1.

Figure 3: Potential $V(\lambda)$ calculated for cubes of size $L=8$ for different values of $W$ (squares) and the analytical fit given in Eq. 21 for indicated values of a and $b=\lambda_{0}^{-1}$.

Figure 4: $P(S)$ for $S=\left(\nu_{2}-\nu_{1}\right) /\left\langle\nu_{2}-\nu_{1}\right\rangle$ corresponding to the same cubes as in Fig 1-2 for $W=4$ (circles), $W=7$ (squares) and Brody distribution with $q=0.7$ (thick line).

Figure 5: $P(S)$ for $S=\left(\nu_{7}-\nu_{6}\right) /\left\langle\nu_{7}-\nu_{6}>\right.$ for $L=6, W=7$ compared to the Wigner surmise.

Figure 6: $P(S)$ for $S=\left(\nu_{2}-\nu_{1}\right) /<\nu_{2}-\nu_{1}>$ for cubes of size $L=8 W=4$ (circles $<\nu_{1}>\approx 2.02$ ) and $W=7$ (Squares $\left\langle\nu_{1}>\approx 10.97\right.$ ) compared with the Wigner surmise for $\beta=1,2$. Applied magnetic field $B=0.25$.

Figure 7: $\Delta_{3}$-statistics as a function of the mean number $n$ of eigenvalues $\lambda_{a}$. Unfolded spectra obtained in a $3 d$-Anderson-model for $W=30$ and $L=7$ at the band centre $(\xi \approx 1)$. The continuous curves are given by $n / 15$ (uncorrelated spectra) and $[\ln (2 \pi * n)+$ $\left.\gamma-5 / 4-\pi^{2} / 8\right] / \pi^{2}$ (random matrix theory).

Figure 8: The inverse localization length as a function of $W-W_{c}$ for $W_{c}=4.5$. Values of $L$ beween 6 and 12 are used to evaluate $\xi$.

Figure 9: average (circles) and variance (diamonds) of $\log (g)$ as a function of $W$ for $3 d$ cubes $(L=8)$.

Figure 10: The first eigenvalues $\nu_{a}$ of a single sample $(L=10)$ at moderate disorder $(W=5.5)$ as a function of the applied magnetic field $B$.

Figure 11: Magnetic field dependence of $\nu_{1}$ for five different samples $(L=10, W=5.5)$.

Figure 12: Magnetic field dependence of $\nu_{1}$ for two samples at different values $W$ $(L=10)$.

Figure 13.a: Fermi energy dependence of the conductance for a single sample deep in the localized regime $(g \ll 1, L=8, W=6, B=0)$.

Figure 13.b: Fermi energy dependence of $g$ near the mobility edge $(L=8, W=6, B=$ $0)$.

Figure 14: Fermi energy dependence of the log-conductance of a single insulating sample $(L=8, W=6)$ for increasing magnetic field $B=0,0.1,0.2, \ldots, 0.5$.

Figure 15: Curve A: $\log (g)$ as a function of the gate voltage (upper coordinate) observed in a mesoscopic GaAs:Si wire (Ref.18) where $g$ has been multiplied by a factor 40 . Curve B: numerical simulation of $\log (g)$ as a function of $E_{F}$ using the $3 d-$-quantum wire network.

Figure 16: Scheme of the network of quantum wires used as a microscopic model. 


\section{References}

[1] E. Abrahams, P. W. Anderson, D. C. Licciardello and T. V. Ramakrishnan, 1979, Phys. Rev. Lett., 42, 673.

[2] F. Wegner, 1979, Z. Phys. B, 35, 207.

[3] For reviews see Mesoscopic Phenomena in Solids (North-Holland, Amsterdam, 1991), ed. by B. L. Althshuler, P. A. Lee and R. A. Webb.

[4] B. L. Altshuler and D. E. Khmelnitskii, 1986, JETP Lett., 42, 359.

[5] S. Feng, P. A. Lee and A. D. Stone, 1987, Phys. Rev. Lett., 56, 1960.

[6] For reviews see Hopping Transport in Solids (North Holland, Amsterdam, 1991), ed. by M. Pollack and B. Shklovskii. (more particularly chapter 9 ).

[7] E. Medina, M. Kardar, Y. Shapir and X. R. Wang, 1989, Phys. Rev. Lett., 62, 941; J. Cook and B. Derrida, 1990, J. of Stat. Phys., 61, 961.

[8] E. Medina and M. Kardar, 1992, Phys. Rev., B 46, 9984.

[9] E. Medina, M. Kardar, Y. Shapir and X. R. Wang, 1990, Phys. Rev. Lett., 64, 1816;

H. L. Zhao, B. Spivak, M. Gelfand and S. Feng, 1991, Phys. Rev., B44, 10760.

[10] E. Medina and M. Kardar, 1991, Phys. Rev. Lett., 66, 3187; Y. Meir, N. S. Wingreen, O. Entin-Wohlman and B. L. Altshuler, 1991, Phys. Rev. Lett, 66, 1517.

[11] V. L. Nguyen, B. Spivak and B. Shklovskii, 1986, JETP Lett., 43, 44.

[12] S. Feng, J.-L. Pichard and F. Zeng, 1993, preprint.

[13] J.-L. Pichard, 1991, in Quantum Coherence in Mesoscopic Systems, (NATO ASI series, Plenum, New York), ed. by B. Kramer; A. D. Stone, P. A. Mello, K. Muttalib and J.-L. Pichard in Ref. 3.

[14] J.-L. Pichard, M. Sanquer, K. Slevin and P. Debray, 1990, Phys. Rev. Lett., 65, 1812 .

[15] S. Feng and J.-L. Pichard, 1991, Phys. Rev. Lett, 67, 753.

[16] P. Hernandez and M. Sanquer, 1992, Phys. Rev. Lett., 68, 1402.

[17] F. Ladieu and J. P. Bouchaud, companion paper submitted to. J. de Physique.

[18] F. Ladieu, D. Mailly and M. Sanquer, companion paper submitted to. J. de Physique.

[19] K. Slevin, J.-L. Pichard and P. A. Mello, 1991, Europhys. Lett, 16, 649.

[20] K. Slevin, J.-L. Pichard and K. Muttalib, 1993, to appear in J. de Physique I.

[21] J.-L. Pichard, N. Zanon, Y. Imry and A.D. Stone, 1989, J. de Physique, 51, 587.

[22] P.A Mello, P. Pereyra and N. Kumar, 1988, Ann. of Phys., 181, 290.

[23] P. Markos and B. Kramer, 1993, preprint.

[24] T. Brody, 1973, Lett. Al Nuovo Cimento, 7, 482.

[25] F. Dyson and M. L. Mehta, 1963, J. of Math. Phys., 4, 701.

[26] O. Bohigas, 1991, in Chaos and Quantum Physics (North Holland, Amsterdam) ed. by M.--J. Giannoni, A. Voros and J. Zinn-Justin.

[27] Muttalib, Chen, Ismail and Nicopoulos, preprint; Blecken and Muttalib, unpublished.

[28] R. Jalabert, J.-L. Pichard and C.W.J. Beenakker, 1993, preprint.

[29] B. Kramer, K. Broderix, A. MacKinnon and M. Schreiber, 1990, Physics, A 167, 163.

[30] A. D. Stone and P. A. Lee, 1985, Phys. Rev. Lett., 54, 1196.

[31] B. L. Altshuler and B. I. Shklovskii, 1986, Sov. Phys. JETP, 64, 127.

[32] B. I. Shklovskii, B. Shapiro, B. R. Sears, P. Lambrianides and H. B. Shore, 1993, to appear in Phys. Rev., B. 
[33] G. Casati, F. Izrailev and L. Molinari, 1990, Phys. Rev. Lett., 64, 1851; M. Feingold, A. Gioletta, F. Izrailev and L Molinari, 1993, to appear in Phys. Rev. Lett.

[34] M. Azbel, 1983, Solid State Commun., 45, 527.

[35] 0 P. Exner and P. Seba, 1989, Reports on Mathematical Physics, 27, 7.

[36] J. E. Avron and L. Sadun, 1989, Phys. Rev. Lett., 62, 3082.

[37] Y. Avishai and Y. B. Band, 1987, Phys. Rev. Lett., 58, 2251

[38] Y. Avishai and J. M. Luck, 1992, Phys. Rev., B 45, 1074.

[39] M. Redheffer, 1962, J. of Math. Phys., 41, 1. 